\title{
Pure Distal 7q Duplication: Describing a Macrocephalic Neurodevelopmental Syndrome, Case Report and Review of the Literature
}

\author{
Kerri Bosfield Jullianne Diaz Eyby Leon \\ Children's National Health System, Rare Disease Institute, Genetics and Metabolism, Washington, DC, USA
}

\section{Established Facts}

- Pure distal $7 \mathrm{q}$ duplications are rarely reported in literature.

- Previous classifications of $7 \mathrm{q}$ duplications are based on the size of the duplication.

\section{Novel Insights}

- Pure distal 7q duplications are a recognizable macrocephalic neurodevelopmental syndrome occurring with a short neck, microretrognathia/palatal anomalies and kyphoscoliosis/skeletal anomalies.

- Newly reported phenotypic features include intermittent dystonic posturing and behavioral irritability.

- Updated review of genes in the region associated with abnormal neurological development and psychological issues that may contribute to the phenotypic findings is given

\section{Keywords}

$7 q$ duplication $\cdot 7 q$ trisomy $\cdot$ Pure $7 q$ duplication $\cdot$ Pure $7 q$ distal duplication - Macrocephalic neurodevelopmental syndrome

\begin{abstract}
Pure distal duplications of $7 q$ have rarely been described in the medical literature. The term pure refers to duplications that occur without an accompanying clinically significant deletion. Pure 7q duplications of various segments have previously been reported in the literature; however, pure distal $7 q$ duplications have only been reported in 21 cases. Twenty of these earlier reports described patients who were identified via karyotype and 1 recently by microarray. Cases have
\end{abstract}

karger@karger.com www.karger.com/msy

(C) 2021 S. Karger AG, Basel

Karger' also been reported in genomic databases such as DECIPHER and the University of California Santa Cruz genome browser. We have reviewed 7 additional cases with distal 7q duplications from these databases and compared them to 7 previously reported distal 7q duplication cases to uncover common features including global developmental delay, frontal bossing, macrocephaly, seizures, kyphoscoliosis/skeletal anomalies, and microretrognathia/palatal anomalies. In this case, we describe a 4-year-old boy with a 30.8-Mb pure duplication of 7q32.1q36.3. Newly reported features associated with this duplication include intermittent dystonic posturing, increased behavioral irritability, eosinophilic esophagitis, segmental vertebral anomalies, and segmental intermittent limb cyanosis. We highlight the importance of using publicly available databases to describe rare genetic syn- 
dromes and to better characterize the features of pure distal $7 q$ duplications and further postulate that duplication of this region represents a recognizable macrocephalic neurodevelopmental syndrome.

(c) 2021 S. Karger AG, Basel

\section{Introduction}

$7 \mathrm{q}$ duplication has long been recognized as a possible multiple congenital anomaly and intellectual disability syndrome, and there have been multiple attempts to postulate a possible phenotype based on the duplicated segment [Courtens et al., 2001]. Novales et al. [1982] separated $7 \mathrm{q}$ duplications into 3 distinct groups by statistical analysis of phenotypic features and breakpoints: Interstitial trisomies of $7(\mathrm{q} 22 \rightarrow \mathrm{q} 31)$, terminal trisomies of 7 (q31 $\rightarrow$ qter), and terminal trisomies of $7(\mathrm{q} 32 \rightarrow \mathrm{qter})$. In this article, we describe these segments as interstitial, proximal and distal, respectively (Fig. 1). Novales et al. [1982] found that low birth weight, developmental delay, low-set ears, small nose, skeletal anomalies, kyphoscoliosis, hypotonicity, absence of microretrognathia, cleft palate, and early postnatal death was common in the distal group. Pure distal duplications of $7 \mathrm{q}$ (those without an accompanying clinically significant deletion) have rarely been described in the medical literature.

In this case, we describe a 4-year-old boy with a pure distal duplication of 7q. Similar duplications have only previously been reported in 21 cases. Common features reported included global developmental delay and dysmorphic features including hypertelorism, micrognathia, low-set and large ears, and macrocephaly. Also reported are neurological deficits such as seizures and brain anomalies, rarely skeletal anomalies, cardiac defects, and genitourinary anomalies [Forabasco et al., 1988; Bartsch et al., 1990; Romain et al., 1990; Verma et al., 1992; Robinet et al., 2000; Rodriguez et al., 2002; Alfonsi et al., 2012]. Scelsa et al. [2008] separated 7q duplications artificially into 4 groups (entire arm, interstitial, proximal, and distal) and suggested that there was no clear genotype-phenotype correlation. However, these categories continue to be helpful when reporting these cases. To further classify these abnormalities, genome browsers have become an essential tool in clinical practice as a way for providers to browse, search, retrieve, and analyze genomic data, especially with underrepresented or rare CNV syndromes [Wang et al., 2013]. This review highlights their viability in the continued understanding of these conditions for patients, families, and providers.

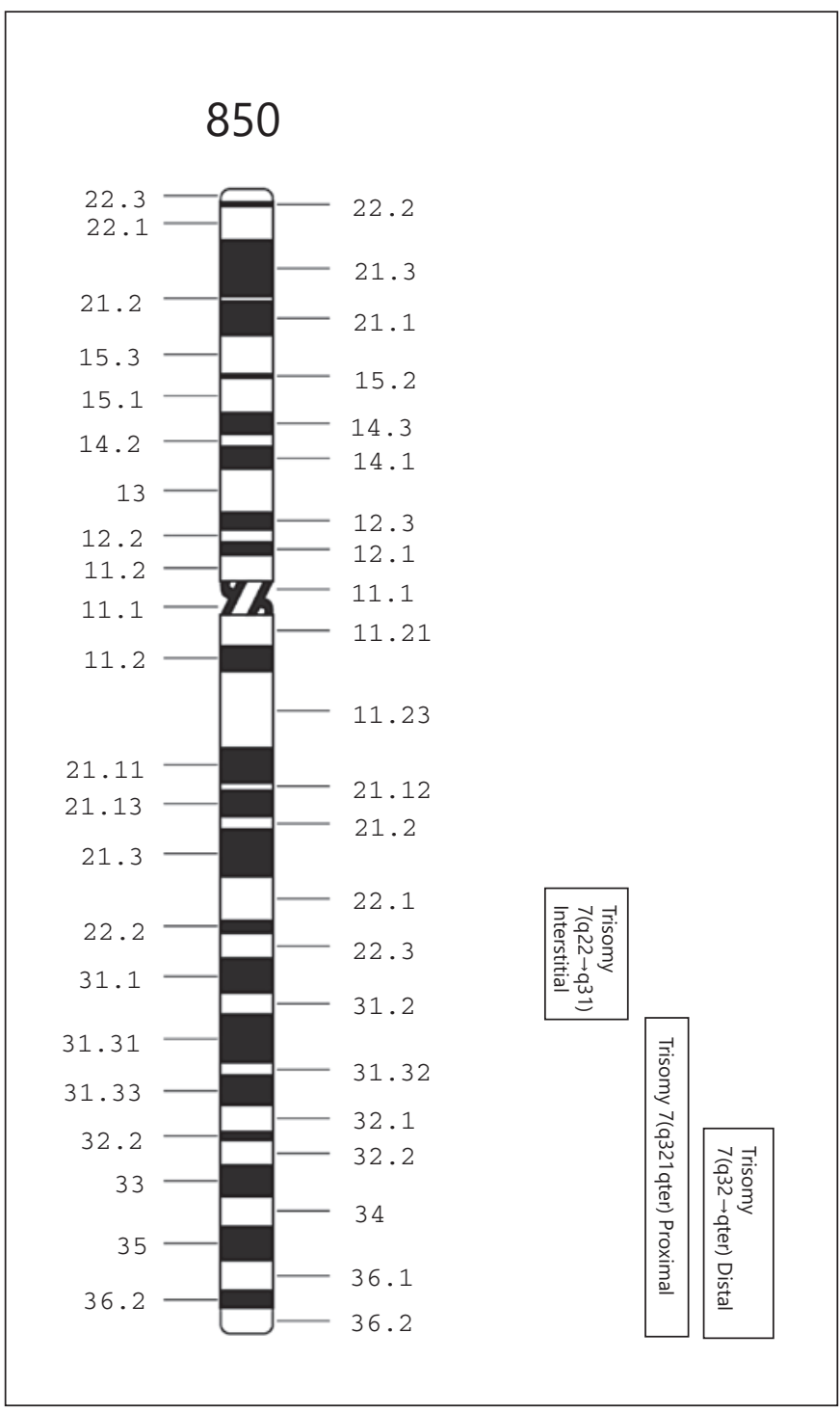

Fig. 1. Chromosome ideogram of chromosome 7 displaying the regions of $7 \mathrm{q}$ duplications presented by Novales et al. [1982].

This is the first review which focuses on the process of reviewing patients with pure distal $7 \mathrm{q}$ duplications from publicly available databases, in order to further describe a possible macrocephalic neurodevelopmental syndrome.

\section{Case Report}

The patient was born at $38^{+4}$ weeks gestational age by normal spontaneous vaginal delivery to a G2P1, 31-year-old mother and 28 -year-old father. Family history was noted for maternal speech delay and attention deficit hyperactivity disorder (ADHD) and paternal ADHD. The couple also shared a daughter who had normal 

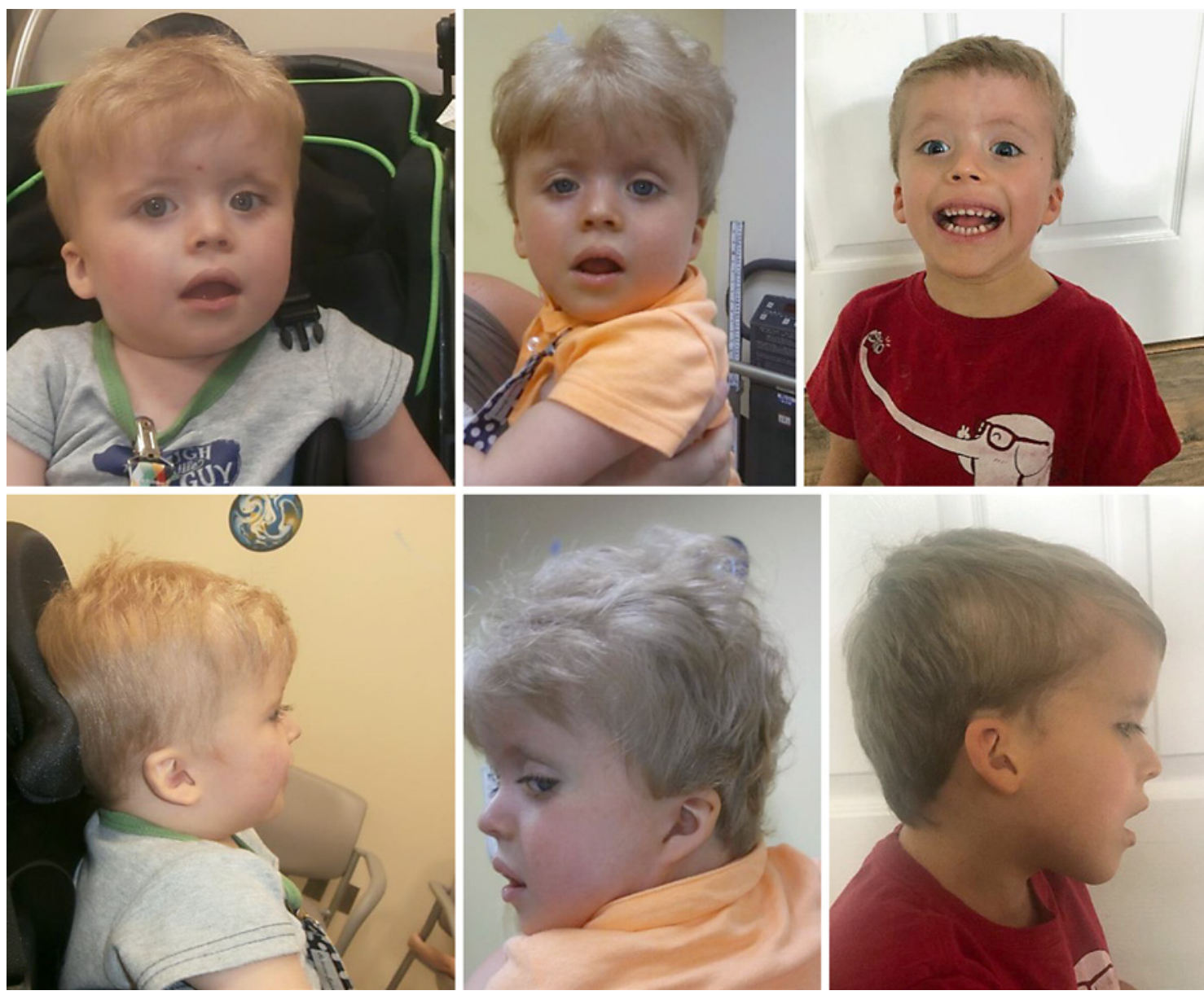

Fig. 2. Patient images at 8,23 , and 45 months. Dysmorphic features include macrocephaly, frontal bossing, high forehead, downslanting palpebral fissures, small pointed nose, depressed nasal bridge, and ear anomalies (overfolded and squared helix, underdeveloped tragus, and prominent anterior crus of antihelix).

growth and development. Both parents were of mixed European ancestry and were nonconsanguineous.

Prenatally on ultrasound, the patient was found to have mild cerebral ventriculomegaly of 11-12 $\mathrm{mm}$ and polyhydramnios with an amniotic fluid index of 35.5 and intraventricular nontraumatic grade II/III hemorrhage.

Birthweight was reported as 3,375 g (50th centile), length 50.8 $\mathrm{cm}$ (50th-90th centile), and head circumference $37 \mathrm{~cm}$ ( $>97$ th centile). He required transfer to the NICU for hypotonia and was briefly intubated for 4 days. He failed his initial newborn hearing screen but passed on repeat prior to discharge on day of life 6 . At 7 weeks of age, he was readmitted for respiratory distress due to aspiration pneumonia secondary to gastroesophageal reflux disease. During that admission, a dysmorphology examination was significant for macrocephaly, frontal bossing, downslanting palpebral fissures, small pointed nose, submucosal cleft palate, low-set posteriorly rotated small ears, and a short neck (Fig. 2). Imaging evaluation showed ventriculomegaly, moderate fenestrated secundum atrial septal defect (ASD) with anterior muscular ventricular septal defect (VSD), bilateral grade 1 hydronephrosis, neuromuscular scoliosis, and cervical/thoracic vertebral segmentation anomalies. At 3 months old, he had a gastrostomy tube placed due to failure to thrive. He would go on to have multiple readmissions for pulmonary issues and was diagnosed with chronic lung disease. At 21 months, he underwent auditory brainstem response testing that was consistent with conductive or possibly mixed hearing loss of mild to moderately-severe degree and started using a bone-anchored hearing aid. However, following his adenoidectomy at 26 months, a repeat auditory brainstem response showed normal hearing, and the use of the hearing aid was discontinued. Severe sleep apnea was initially thought to be central, but a sleep study revealed it to be more obstructive requiring continuous positive airway pressure/bilevel positive airway pressure which resolved after adenoidectomy and myringotomy tube placement. Other medical problems included feeding difficulties, alternating exotropia, mild myopia, multiple rib fractures secondary to osteopenia of unknown etiology, intermittent dystonic posturing, eosinophilic esophagitis, and segmental intermittent cyanosis of the left upper limb of unknown etiology. Seizures began at 31 months, which were classified as complex febrile and focal aware epilepsy and have been well controlled on levetiracetam. An EEG obtained after seizure onset showed no clinical or subclinical seizures, but did 


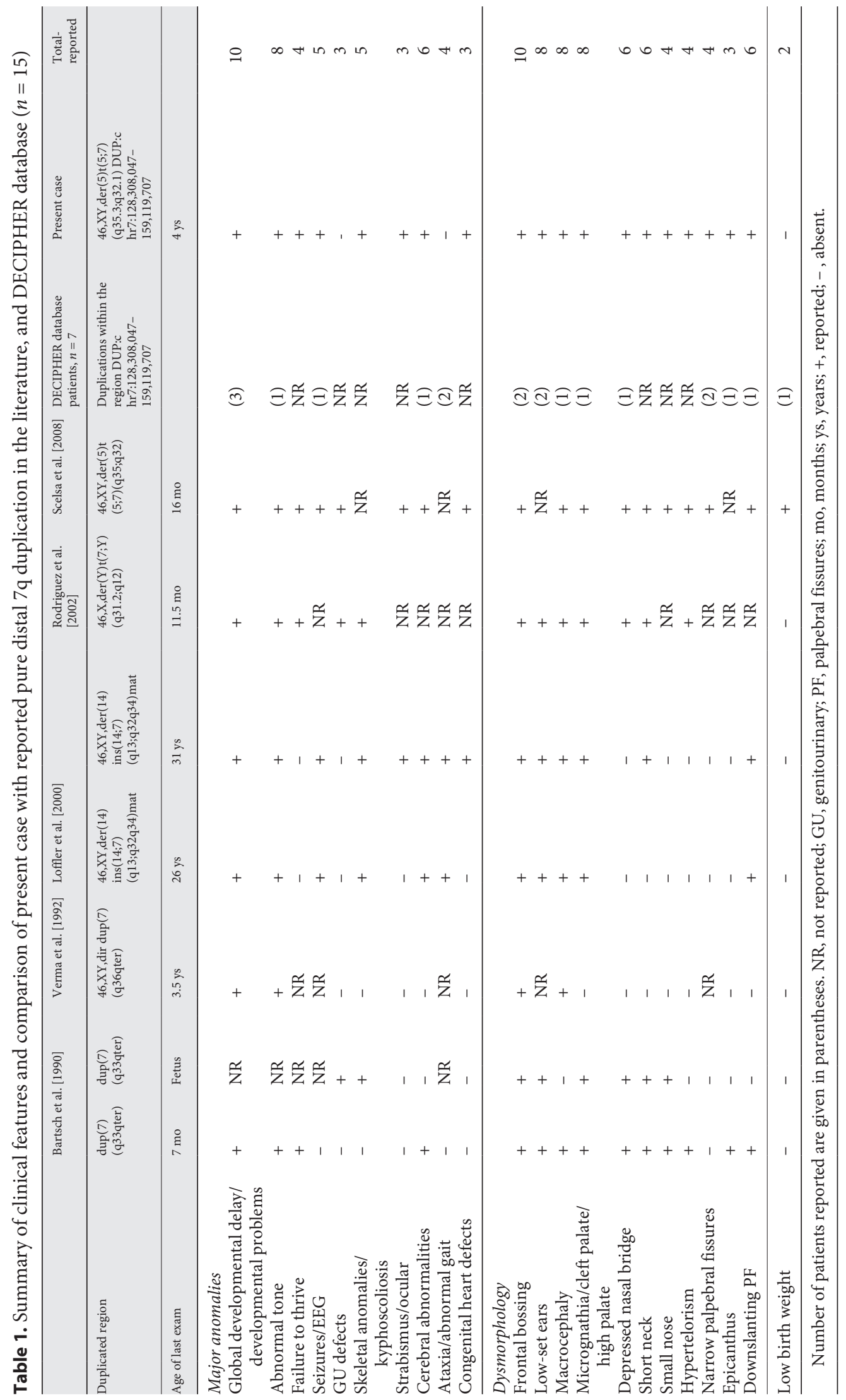


demonstrate mild generalized slowing with superimposed bilateral occipital slowing (right $>$ left).

The boy displayed global developmental delays affecting gross motor, fine motor, receptive/expressive language, and cognitive skills. His early years of development were tracked closely and demonstrated slow gains in acquiring skills, but small improvements with various therapies. Standardized developmental testing at 32 months revealed a cognitive skill level of approximately 11-16 months, a receptive language level of 13 months, and expressive language at 15 months. It is also thought that chronic otitis media with effusion also contributed to his early delay of speech development. At 36 months, he was attending a structured childhood education program where he received an individualized education plan.

During his last physical examination at 40 months, growth parameters included weight $14.5 \mathrm{~kg}$ (38th percentile), height $97.5 \mathrm{~cm}$ (47th percentile), and head circumference $54 \mathrm{~cm}$ ( $>99$ th percentile).

Chromosomal microarray analysis was performed in the immediate newborn period secondary to multiple congenital anomalies. This identified a 30.8-Mb duplication at 7q32.1q36.3, arr[hg19] 7q32.1q36.3(128,308,047-159,119,707)×3.

A standard karyotype was done to further characterize the duplication found on the chromosomal microarray which showed a structural abnormality of the long arm of chromosome 5 secondary to an unbalanced translocation between chromosomes 5 and 7, 46,XY,der(5)t(5;7)(q35.3;q32.1). The maternal karyotype was normal; paternal karyotyping was not performed. Thus, it is unknown whether this chromosome abnormality is de novo or due to a paternal balanced translocation.

The prior chromosomal microarray study did not reveal a terminal deletion of $5 \mathrm{q}$, and it was assumed that the chromosome 5 breakpoint was in or very near the telomeric repeat region which did not include any clinically relevant genes. From a diagnostic perspective, the abnormality was considered to be a pure duplication of distal 7q.

Trio whole-exome sequencing was performed at parental request given concerns for chronic irritability and suspected pain symptoms that could not otherwise be explained. This testing did not show pathogenic or likely pathogenic variants, but demonstrated several variants of unknown clinical significance. The variants were determined to be noncontributory on a clinical and molecular bases. The variants were interpreted according to the ACMG guidelines. For dominant disorders (ITPR1, MARS, NALCN, ACAN, $K M T 2 B)$, the variants in the genes were inherited from a parent. For recessive disorders ( $A L X 3, C R B N, I T G A 7)$, a second variant was not detected in these genes. The variant in the $C R B N$ gene was the only de novo variant and was predicted to be benign.

\section{Methods}

\section{Patient Search Process}

Various genome browsers were used during our search for other reported individuals with pure distal $7 \mathrm{q}$ duplications. The University of California Santa Cruz (UCSC) genome browser [Karolchik et al., 2004] was used to locate our patient's duplicated region in chromosome 7 using genome assembly GRCh37(hg19). We were then able to alter the tracks of the platform to locate all DECIPHER (Database of genomic variation and Phenotype in $\mathrm{Hu}-$ mans using Ensembl Resources) [Firth et al., 2009] CNVs in this region. A total of 135 reported individuals with distal $7 q$ duplications, included within the region of our case were identified, and their reported clinical features and duplication size were noted. Through the UCSC genome browser, we were redirected to the DECIPHER database for each patient.

DECIPHER was used to identify 72 out of 135 patients reported in the UCSC genome browser that had pure distal 7q duplications. Once these 72 patients were identified, we then used the UCSC genome table browser to identify all NCBI RefSeq genes located in each duplicated region [Pruitt et al., 2005]. These genes were then compared to identify commonly shared genes in each region.

The Franklin database by Genoox (https://franklin.genoox. com/home), a variant interpretation tool was used to analyze each duplication. The database uses evidence aggregated from public databases and utilizes the ACMG/ClinGen proposed guidelines to suggest classifications of a queried CNV gain or loss [Riggs et al., 2020]. Out of the 72 duplications identified during this process, only those classified as pathogenic, 7 total, were included in this review. This number of patients was compared to the 7 published cases along with the present case, giving a total of 15 patients with distal $7 \mathrm{q}$ duplications (Table 1).

\section{Results}

Table 1 summarizes the most commonly reported features from our literature and database searches. Pure distal $7 \mathrm{q}$ duplications appear to possess distinctive phenotypic characteristics, the majority of which appear to be a neurodevelopmental phenotype. Initial reports in the literature of patients with pure distal $7 \mathrm{q}$ duplications suggested the following distinctive craniofacial features: macrocephaly, frontal bossing, hypertelorism, small nose, depressed nasal bridge, micrognathia, cleft lip/palate, epicanthus, and malformed low-set ears. Further review of the DECIPHER database confirmed some of these characteristic features. Most interestingly, macrocephaly and frontal bossing appear to be the 2 most common dysmorphic features found in these patients reported 10 and 8 times, respectively. Additionally, lowset ears, palatal anomalies, and a short neck were also a common occurrence reported in 8,8 , and 6 patients, respectively. Developmental delay (particularly speech impairment) and intellectual disability were the most commonly identified features of pure distal 7q duplication patients with 10 of the reported patients, although the degree of delay or intellectual impairment was not clarified for each patient. There were individual reports of various neurological features, and collectively, they represented a significant proportion of the morbidity in this population. The neurological abnormalities included abnormal tone in 8 patients, seizures/EEG abnormalities in 5 patients, ataxia/abnormal gait in 4 patients, and brain abnormalities in 6 patients (dilation of lateral ven- 
Table 2. Potential candidate OMIM genes found in our patient's duplicated region. None have been proven to have triplosensitivity.

\begin{tabular}{|c|c|}
\hline Gene & Function \\
\hline CNTNAP2 & $\begin{array}{l}\text { This gene encodes a member of the neurexin family which functions in the vertebrate nervous system as cell adhesion molecules and } \\
\text { receptors. This protein is localized at the juxtaparanodes of myelinated axons, and mediates interactions between neurons and glia } \\
\text { during nervous system development and is also involved in localization of potassium channels within differentiating axons. This gene } \\
\text { has been implicated in multiple neurodevelopmental disorders, including Gilles de la Tourette syndrome, schizophrenia, epilepsy, } \\
\text { autism, ADHD, and intellectual disability [Provided by RefSeq, Jul 2017]. }\end{array}$ \\
\hline GALNTL5 & $\begin{array}{l}\text { Membrane-bound polypeptide N-acetylgalactosaminyltransferases catalyze the first step in mucin-type O-glycosylation of peptides in } \\
\text { the Golgi apparatus. O-glycosylation of NOTCH1 leading to the activation of the Notch signaling pathway [Al Dhaibani et al., 2017] }\end{array}$ \\
\hline GALNT11 & $\begin{array}{l}\text { Membrane-bound polypeptide N-acetylgalactosaminyltransferases catalyze the first step in mucin-type O-glycosylation of peptides in } \\
\text { the Golgi apparatus. O-glycosylation of NOTCH1 leading to the activation of the Notch signaling pathway [Al Dhaibani et al., 2017] }\end{array}$ \\
\hline PAXIP1 & $\begin{array}{l}\text { This gene is a member of the paired box (PAX) gene family and encodes a nuclear protein with six BRCT (breast cancer carboxy- } \\
\text { terminal) domains. This protein plays a critical role in maintaining genome stability, condensation of chromatin, and progression } \\
\text { through mitosis, [Provided by RefSeq, Jul 2008] shows regional specificity for the cerebellum (HumanProtien Atlas) }\end{array}$ \\
\hline PTPRN2 & $\begin{array}{l}\text { Protein tyrosine phosphatase receptor type } \mathrm{N} \text { polypetide, which is implicated in the development of the nervous system and pancreatic } \\
\text { endocrine cells, selectively expressed in neurons and glia of the brain and a few other tissues [Cui et al., 1996], candidate susceptibility } \\
\text { gene for ADHD [Lionel et al., 2011] }\end{array}$ \\
\hline SHH & $\begin{array}{l}\text { Encodes a protein that is instrumental in patterning the early embryo. It has been implicated as the key inductive signal in patterning of } \\
\text { the ventral neural tube, the anterior-posterior limb axis, and the ventral somites. Defects in this protein or in its signaling pathway are a } \\
\text { cause of holoprosencephaly, mutations in a long range enhancer located approximately } 1 \text { mega base upstream of this gene disrupt limb } \\
\text { patterning and can result in preaxial polydactyly [Provided by RefSeq, Jul 2008]. }\end{array}$ \\
\hline
\end{tabular}

tricles and subarachnoid space and cisterns, cerebellar hypoplasia, and ventriculomegaly). Skeletal abnormalities have been reported as a feature of $7 \mathrm{q}$ duplications, in particular trisomies 7(q31qter) and 7(q32qter) [Novales et al., 1982]. This author also postulated that kyphoscoliosis was more suggestive of trisomies 7(q31qter). Kyphoscoliosis was reported in 4 patients, including the present case, and other skeletal anomalies reported previously include missing ribs and delayed ossification of phalanges and upper cervical vertebrae [Bartsch et al., 1990]. Ocular issues such as high-grade myopia, astigmatism and sclerosis of the fundi and strabismus were also reported in 3 cases [Loffler et al., 2000]. Our patient was noted to have a moderate fenestrated secundum ASD and anterior muscular VSD; however, cardiovascular disease was not a common occurrence in this group and was reported in only 2 other patients includ- ing mild aortic insufficiency [Loffler et al., 2000] and pulmonary valve dysplasia, intraventricular septal defect, and intra-atrial septal defect [Scelsa et al., 2008]. Low birth weight, even though reported as a common finding, was only reported in 2 patients [Novales et al., 1982; Scelsa et al., 2008].

\section{Discussion}

Given that developmental delays and macrocephaly appear to be 2 of the common features of pure distal $7 \mathrm{q}$ duplications, we suggest that this diagnosis represents a recognizable macrocephalic neurodevelopmental syndrome that occurs with a short neck, microretrognathia/ palatal anomalies, and kyphoscoliosis/skeletal anomalies. However, differing from a previous report by Novales et 
al. [1982], low birth weight was not a common occurrence.

The smallest duplication found was 2,116 bp and the largest was 24,911,756 bp. Larger duplications usually include more protein-coding RefSeq genes and subsequently will be classified as likely pathogenic or pathogenic, which is an argument for the usefulness of reporting the size and region of duplications. Pathogenic distal 7q duplications did not appear to have recurrent breakpoints. Additionally, section 3 of the newly proposed ACMG and ClinGen technical standards for the interpretation and reporting of constitutional CNVs, considers the number of protein-coding RefSeq genes wholly or partially included in the copy-number gain.

Our review showed large duplications with breakpoints within regions of known protein-coding genes. However, these CNVs were classified as uncertain significance which is usually the case with copy number gains due to the lack of true triplosensitivity studies that can reveal the consequences of these duplications.

The genes located within our patient's duplication region and 7 other DECIPHER patients were assessed for similarities and differences for a potential critical region. Potential candidate genes found in our patient's duplicated region, along with their function, are shown in Table 2 .

The most common OMIM (https://omim.org/) gene noted among these patients was $L M B R 1$ (6/8). Duplications of this gene have not been directly linked to any genetic condition. However, alterations of an $\mathrm{SHH}$ regulatory element (ZRS) that resides in intron 5 of the $L M B R 1$ gene have been associated with preaxial polydactyly II, triphalangeal thumb-polysyndactyly syndrome, and isolated triphalangeal thumb [Lettice et al., 2003; Gurnett et al., 2007; Furniss et al., 2008; Semerci et al., 2009]. Duplications of $L M B R 1$ can potentially alter $S H H$ expression, although this has not been proven. One out of 7 DECIPHER patients was reported to have hand polydactyly and 1-5 finger syndactyly. Although several of the OMIM genes have sufficient evidence for reported haploinsufficiency, there is little data on triplosensitivity of the genes in this region. For example, The ClinGen Resource [Rhem et al., 2015] references a 2009 report of a maternally inherited duplication encompassing $\mathrm{SHH}$ in a patient with semilobar holoprosencephaly; however, the functional consequence of this duplication remains unknown [Bendavid et al., 2009]. Another case report by Kroeldrup et al. [2012] describes a patient with congenital muscular hypertrophy, associated with a $0.3-\mathrm{Mb}$ duplication of $7 \mathrm{q} 36.3$ which included the $S H H$ and $R B M 33$ genes. These authors postulated that overexpression of SHH might help to explain the muscular hypertrophy; however, the extent of RBM33 involvement could not be assessed, and none of the reported patients showed muscular hypertrophy. Micale et al. [2017] described 2 discontinuous maternally inherited 7q36.3 microduplications with 1 duplication also including $\mathrm{SHH}$ in a fetus. The phenotype included multiple congenital anomalies with a right-sided heart position, absent left kidney, absent right radius and thumb, fixed immobile right forearm, wrist, and digits, at least 1 hemivertebrae, and scoliosis of the sacral spine. These 2 case studies further exemplify how duplications involving the $\mathrm{SHH}$ gene and other regulatory elements can have large phenotypic consequences.

Haploinsufficiency of $S H H$ along with MNX1 have been hypothesized to play a role in embryological maldevelopment of vertebral and rib anomalies. This is particularly a feature of Currarino syndrome [Gupta et al., 2014]. Individuals with Currarino syndrome may have deletions of 7q36 and experience defects of the sacral vertebrae. Gupta et al. [2014] note that interaction between $\mathrm{SHH}$ and noggin genes produced by the notochord along with the ventromedial portion of the embryological somite leads to differentiation of sclerotomes to form vertebrae. One can postulate that overexpression of these genes can also be implicated in the skeletal and vertebral anomalies noted in our cohort; however, this has not been proven. A duplication of MNX1 was noted in 5/9 studied patients.

In a more recent article by Pavone et al. [2019], the authors described a child with a partial duplication of $7 q 31.32(122,254,792-122,376,908)$ with dysmorphic features, ASD, moderate intellectual disability, and epilepsy. Although the authors described the dysmorphic features as mild, the child in the report shared similar features to our patient including macrocephaly and seizures. This child harbored a more proximal duplication compared to our cohort, and the authors postulated that rearrangements of the AUTS1 locus, which included CADPS2, RNF133 and RFN148, were partly responsible for the patient's neurological and behavioral findings due to disruption of cerebral development. These genes were not located within our patient's duplicated region. Al Dhaibani et al. [2017] reported a child with a de novo chromosome $7 \mathrm{q} 36.1 \mathrm{q} 36.2$ triplication with distinctive facial features, similar to our investigated cohort and multiple congenital anomalies. The GALNT11 gene was found in the duplicated region in 3 out of our 8 reviewed patients. The authors of this report postulated that GALNT11 en- 
codes a membrane-bound polypeptide $\mathrm{N}$-acetylgalactosaminyltransferase that can O-glycosylate NOTCH1 leading to the activation of the Notch signaling pathway, explaining the pathogenicity of $7 \mathrm{q} 36$ triplication. They concluded that this hypothesis would have to be further investigated.

Another gene duplicated in our reviewed patients was CASP2 noted in 4/8 patients. CASP2 has been shown to have an important role in the developing human neocortex and is needed for normal cognitive ability [Carlsson et al., 2011; Di Donato et al., 2016]. In addition, inhibition of this gene reduces hypoxic-ischemia and excitotoxic neonatal brain injury. One possibility is that upregulation can potentially have a consequence by increasing the risk of neonatal brain injury in these fragile patients leading to some of the reported neurological complications. Further studies would have to be undertaken to support this hypothesis.

VIPR 2 which was duplicated in $5 / 8$ patients encodes a peptide which is highly expressed in the brain where it acts as a neurotransmitter inducing cAMP activity. Firouzabadi et al. [2017] suggested that duplications of this gene, which was found in an autistic father and son, may suggest evidence to support the role in neuropsychiatric phenotypes and ADHD.

The distal region of $7 \mathrm{q}$ also contain genes that are related to ephs and ephrin receptors and neurite growthpromoting factors. These genes (EPHA1, EPHB6, and $P T N)$ play an important role in axonal guidance and migration in the developing and adult brain [Rodger et al., 2012]. Deficiency of PTN or pleiotrophin has been shown in mice to cause coordination impairment and disruption of establishing proper functional cerebellar circuits [Hamza et al., 2016]. Proximal to our patient's duplication, at 7q31, exists a metabotropic glutamate receptor (mGluR) gene GRM8 that may be related to neurodevelopmental disorders, autism and severe intellectual disability [Sangu et al., 2017]. It is suspected that increased expression of functional mGluR may trigger the development of seizure activity [Al-Ghoul et al., 1998; Loffler et al., 2000]. Further studies of these duplications may investigate whether inclusion of the GRM8 gene in $7 q$ duplications may modify the phenotypic presentation of intellectual disabilities and seizures in these patients.

\section{Limitations}

After reviewing 135 patients with distal 7q duplications, we were only able to include 7 total with pathogenic variants in this review. Of note, some of the pa- tients that were not included in the review displayed various behavioral and neurodevelopmental phenotypic features; however, they were not included because their duplications were classified as unknown significance. We also realize that public databases are user dependent and rely on subjective reporting by the individual contributing to the database which can limit the cohort that is reviewed. Additionally, phenotypic terminology can vary between clinical professionals. Previous literature reviewed for patients with pure distal $7 \mathrm{q}$ duplications was also limited by terminology that today would be considered inappropriate, in addition they lacked microarray data, and therefore possible microduplications and deletions could not be excluded on their chromosome analysis results alone.

\section{Conclusion}

Pure distal 7q duplication can be considered a recognizable macrocephalic neurodevelopmental syndrome that may have increased presentation of kyphoscoliosis/skeletal anomalies and microretrognathia/palatal anomalies compared to other $7 \mathrm{q}$ duplications. This review demonstrates the importance of publicly shared genomic databases to further unify features of this duplication syndrome. In the long term, these databases should also focus on reporting rare pure duplication syndromes separate from those with accompanying deletions, so that we are better able to identify features of the duplicated regions, which still require much exploration.

\section{Acknowledgement}

This study makes use of data generated by the DECIPHER community. A full list of centers who contributed to the generation of the data is available from http://decipher.sanger.ac.uk and via email from decipher@sanger.ac.uk.

\section{Statement of Ethics}

The research presented in the manuscript was conducted ethically in accordance with the World Medical Associated Declaration of Helsinki and the appropriate guidelines for human studies. Written informed consent and permission to publish the case and images were obtained from the patient's guardian. IRB approval was not required for publication of this single case study. 


\section{Conflict of Interest Statement}

The authors declare no potential conflicts of interest with respect to the research, authorship and or publication of this article.

\section{Funding Sources}

Funding for the project was provided by Wellcome.

\section{Author Contributions}

Kerri Bosfield devised the main conceptual ideas for the project, literature review, analysis of the results, creation of figures, and writing of the manuscript. Jullianne Diaz contributed with the literature review, writing, and editing of the manuscript. Eyby Leon supervised and contributed to the writing of the manuscript. All authors discussed the results and contributed to the final manuscript.

\section{References}

Al Dhaibani MA, Allingham-Hawkins D, ElHattab AW. De novo chromosome $7 \mathrm{q} 36.1 \mathrm{q} 36.2$ triplication in a child with developmental delay, growth failure, distinctive facial features, and multiple congenital anomalies: a case report. BMC Med Genet. 2017; 18(1): 118

Alfonsi M, Palka C, Morizio E, Gatta V, Franchi S, Guanciali Franchi P, et al. A new case of pure partial $7 \mathrm{q}$ duplication. Cytogenet $\mathrm{Ge}-$ nome Res. 2012;136(1):1-5.

Al-Ghoul WM, Meeker RB, Greenwood RS. Kindled seizures increase metabotropic glutamate receptor expression and function in the rat supraoptic nucleus. J Neurosci Res. 1998 54(3):412-23

Bartsch O, Kalbe U, Ngo TK, Lettau R, Schwinger E. Clinical diagnosis of partial duplication 7q. Am J Med Genet. 1990;37(2):254-7.

Bendavid C, Rochard L, Dubourg C, Seguin J, Gicquel I, Pasquier L, et al. Array-CGH analysis indicates a high prevalence of genomic rearrangements in holoprosencephaly: an updated map of candidate loci. Hum Mutat. 2009;30(8):1175-82.

Carlsson Y, Schwendimann L, Vontell R, Rousset CI, Wang X, Lebon S, et al. Genetic inhibition of caspase-2 reduces hypoxic-ischemic and excitotoxic neonatal brain injury. Ann Neurol. 2011;70(5):781-9.

Courtens W, Vroman S, Vandenhove J, Wiedemann U, Schinzel A. Pre- and perinatal findings in partial trisomy $7 \mathrm{q}$ resulting from balanced parental translocations $\mathrm{t}(7 ; 21)$ and $\mathrm{t}(4$; 7). Prenat Diagn. 2001;21(8):642-8.

Cui L, Yu WP, DeAizpurua HJ, Schmidli RS, Pallen CJ. Cloning and characterization of islet cell antigen-related protein-tyrosine phosphatase (PTP), a novel receptor-like PTP and autoantigen in insulin-dependent diabetes. J Biol Chem. 1996;271(40):24817-23.

Di Donato N, Jean YY, Maga AM, Krewson BD, Shupp AB, Avrutsky MI, et al. Mutations in CRADD Result in Reduced Caspase-2-Mediated Neuronal Apoptosis and Cause Megalencephaly with a Rare Lissencephaly Variant. Am J Hum Genet. 2016;99(5): 1117-29.

Firouzabadi SG, Kariminejad R, Vameghi R, Darvish $\mathrm{H}$, Ghaedi $\mathrm{H}$, Banihashemi S, et al. Copy Number Variants in Patients with Autism and Additional Clinical Features: Report of
VIPR2 Duplication and a Novel Microduplication Syndrome. Mol Neurobiol. 2017;54(9): 7019-27.

Firth HV, Richards SM, Bevan AP, Clayton S, Corpas M, Rajan D, et al. DECIPHER: Database of Chromosomal Imbalance and Phenotype in Humans Using Ensembl Resources. Am J Hum Genet. 2009;84(4):524-33.

Forabosco A, Baroncini A, Dalpra L, Chessa L, Giannotti A, Maccagnani F, et al. The phenotype of partial dup $(7 \mathrm{q})$ reconsidered: a report of five new cases. Clin Genet. 1988;34(1):4859.

Furniss D, Lettice LA, Taylor IB, Critchley PS, Giele $\mathrm{H}$, Hill RE, et al. A variant in the sonic hedgehog regulatory sequence (ZRS) is associated with triphalangeal thumb and deregulates expression in the developing limb. Hum Mol Genet. 2008;17(16):2417-23.

Gupta R, Sharma SB, Mathur P, Goyal RB. Variants of Currarino Syndrome: Embryological Association and Review of Pertinent Literature. Int J Embryol. 2014;2014:1.

Gurnett CA, Bowcock AM, Dietz FR, Morcuende JA, Murray JC, Dobbs MB. Two novel point mutations in the long-range $\mathrm{SHH}$ enhancer in three families with triphalangeal thumb and preaxial polydactyly. Am J Med Genet A. 2007;143A(1):27-32.

Hamza MM, Rey SA, Hilber P, Arabo A, Collin T, Vaudry D, et al. Early Disruption of Extracellular Pleiotrophin Distribution Alters Cerebellar Neuronal Circuit Development and Function. Mol Neurobiol. 2016;53(8):520316.

Karolchik D, Hinrichs AS, Furey TS, Roskin KM, Sugnet CW, Haussler D, et al. The UCSC Table Browser data retrieval tool. Nucleic Acids Res. 2004;32(Database issue):D493-6.

Kroeldrup L, Kjaergaard S, Kirchhoff M, Kock K, Brasch-Andersen C, Kibaek M, et al. Duplication of $7 \mathrm{q} 36.3$ encompassing the Sonic Hedgehog ( $\mathrm{SHH})$ gene is associated with congenital muscular hypertrophy. Eur J Med Genet. 2012;55(10):557-60.

Lettice LA, Heaney SJ, Purdie LA, Li L, de Beer P, Oostra BA, et al. A long-range Shh enhancer regulates expression in the developing limb and fin and is associated with preaxial polydactyly. Hum Mol Genet. 2003;12(14):1725-35.

Lionel AC, Crosbie J, Barbosa N, Goodale T, Thiruvahindrapuram B, et al. Rare copy num- ber variation discovery and cross-disorder comparisons identify risk genes for ADHD. Sci Transl Med. 2011;3(95):95ra75.

Loffler J, Utermann B, Duba HC, Mayr U, Utermann G, Erdel M. Mental and psychomotoric retardation in two brothers with pure partial trisomy 7q32-q34 due to a maternal insertion (14;7). Am J Med Genet. 2000;91(4):291-7.

Meng E, Shevde LA, Samant RS. Emerging roles and underlying molecular mechanisms of DNAJB6 in cancer. Oncotarget. 2016;7(33): 53984-96.

Micale M, Embrey B, Hubbell K, Beaudry-Rogers K, Whitten A. Prenatal identification of two discontinuous maternally inherited chromosome $7 \mathrm{q} 36.3$ microduplications totaling 507 $\mathrm{kb}$ including the sonic hedgehog gene in a fetus with multiple congenital anomalies. Clin Case Rep. 2017;5(6):993-9.

Novales MA, Fernandez-Novoa C, Hevia A, San Martin V, Galera H. Partial trisomy for the long arm of chromosome 7. Case report and review. Hum Genet. 1982;62(4):378-81.

Pavone P, Corsello G, Marino SD, Ruggieri M, Falsaperla R. 7q31.32 partial duplication: First report of a child with dysmorphism, autistic spectrum disorder, moderate intellectual disability and, epilepsy. Literature review. Epilepsy Res. 2019;158:106223.

Pruitt KD, Tatusova T, Maglott DR. NCBI Reference Sequence (RefSeq): a curated non-redundant sequence database of genomes, transcripts and proteins. Nucleic Acids Res. 2005; 33(Database issue):D501D504.

Rehm HL, Berg JS, Brooks LD, Bustamante CD, Evans JP, Landrum MJ, et al. ClinGen--the Clinical Genome Resource. N Engl J Med. 2015;372(23):2235-42.

Riggs ER, Andersen EF, Cherry AM, Kantarci S, Kearney H, Patel A, et al. Technical standards for the interpretation and reporting of constitutional copy-number variants: a joint consensus recommendation of the American College of Medical Genetics and Genomics (ACMG) and the Clinical Genome Resource (ClinGen). Genet Med. 2020; 22(2):245-57.

Robinet C, Douvier S, Khau Van Kien P, Favre B, Luquet I, Nadal N, et al. Prenatal diagnosis of a partial trisomy $7 \mathrm{q}$ in two fetuses with bilateral ventriculomegaly. Prenat Diagn. 2000; 20(11):936-8. 
Rodger J, Salvatore L, Migani P. Should I stay or should I go? Ephs and ephrins in neuronal migration. Neurosignals. 2012;20(3):190-201.

Rodriguez L, Lopez F, Paisan L, de la Red Mdel M, Ruiz AM, Blanco M, et al. Pure partial trisomy 7q: two new patients and review. Am J Med Genet. 2002;113(2):218-24.

Romain DR, Cairney H, Stewart D, ColumbanoGreen LM, Garry M, Parslow MI, et al. Three cases of partial trisomy 7q owing to rare structural rearrangements of chromosome 7. J Med Genet. 1990;27(2):109-13.
Sangu N, Shimojima K, Takahashi Y, Ohashi T, Tohyama J, Yamamoto T. A 7q31.33q32.1 microdeletion including LRRC4 and GRM8 is associated with severe intellectual disability and characteristics of autism. Hum Genome Var. 2017;4:17001.

Scelsa B, Bedeschi FM, Guerneri S, Lalatta F, Introvini P. Partial trisomy of 7q: case report and literature review. J Child Neurol. 2008; 23(5):572-9.
Semerci CN, Demirkan F, Ozdemir M, Biskin E, Akin B, Bagci H, et al. Homozygous feature of isolated triphalangeal thumb-preaxial polydactyly linked to 7q36: no phenotypic difference between homozygotes and heterozygotes. Clin Genet. 2009;76(1):85-90.

Verma RS, Conte RA, Pitter JH. Tandem duplication of the terminal band of the long arm of chromosome 7 (dir dup (7)(q36 $\rightarrow$ qter)). J Med Genet. 1992;29(5):344-5.

Wang J, Kong L, Gao G, Luo J. A brief introduction to web-based genome browsers. Brief Bioinform. 2013;14(2):131-43. 